\title{
Tunneling of the Closed Friedmann Universe with Generation of Scalar Waves
}

\author{
V. Ts. Gurovich*, H.-J. Schmidt ${ }^{\dagger}$ and I. V. Tokareva ${ }^{\ddagger}$
}

October 29, 2018

\begin{abstract}
The evolution of the closed Friedmann Universe with a packet of short scalar waves is considered with the help of the Wheeler-DeWitt equation. The packet ensures conservation of homogeneity and isotropy of the metric on average. It is shown that during tunneling the amplitudes of short waves of a scalar field can increase catastrophically promptly if their influence to the metric do not take into account. This effect is similar to the Rubakov-effect of catastrophic particle creation calculated already in 1984 .

In our approach to the problem it is possible to consider self-consistent dynamics of the expansion of the Universe and amplification of short waves. It results in a decrease of the barrier and interruption of amplification of waves, and we get an exit of the wave function from the quantum to the classically available region.
\end{abstract}

\section{Statement of the problem}

Here we present the basic equations of the problem.

In the space-time of the closed cosmological Friedmann models with space-time metric

$$
d s^{2}=d t^{2}-a^{2}(t)\left[d \chi^{2}+\sin ^{2} \chi\left(d \theta^{2}+\sin ^{2} \theta d \varphi^{2}\right)\right],
$$

where $0 \leq \chi, \theta \leq \pi, \quad 0 \leq \varphi \leq 2 \pi$, the action is assumed to be

$$
S=\int\left[-\frac{R}{16 \pi G}-\bar{\Lambda}+\frac{1}{2} \varphi^{, i} \varphi_{, i}-\frac{1}{2} m^{2} \varphi^{2}\right] \sqrt{-g} d \Omega,
$$

Here $R$ is a scalar curvature, $\varphi$ is a potential of the scalar quantum field with a mass $m$, $(\hbar=c=1), \bar{\Lambda}$ is a cosmological $\Lambda$ - term.

The equation for $\varphi$ has the form $\square \varphi+m^{2} \varphi=0$. Below let us set the scalar field as radial standing waves with time-dependent amplitudes

$$
\varphi_{k}=\mathrm{A}_{k}(t) f_{k}(\chi) ; \quad f_{k}(\chi)=\frac{\sin k \chi}{\sin \chi}
$$

${ }^{*}$ Physics Institute of NAN KR, 265 a, Chui str., Bishkek, 720071, Kyrgyz Republic, e-mail gurovich@grav.freenet.bishkek.su

†Potsdam Univ., Inst. f. Math., Germany and Inst. f. Theoretische Physik, Freie Univ. Berlin, Germany, hjschmi@rz.uni-potsdam.de

${ }^{\ddagger}$ KRSU, 44, Kievskaya st., Bishkek, 720000, Kyrgyz Republic, e-mail astra@freenet.kg 
Note that the functions $f_{k}(\chi)$ do not have singularities on the poles $(\chi=0, \pi)$ for integer numbers $k$, and that they are orthonormal on the coordinate $\chi$ in the measure defined by metric (1), i.e.,

$$
\int \sin ^{2} \chi f_{k} f_{n} d \chi=\delta_{k n}
$$

Now the action (2) can be integrated over the angular variables, and we rewrite it after replacement of $R$ by its value through $a$ as

$$
S=\frac{3 \pi}{4 G} \int\left[\sum_{k}\left(a^{3} \dot{\mathrm{A}}_{k}^{2}-k^{2} a \mathrm{~A}_{k}^{2}-m^{2} a^{3} \mathrm{~A}_{k}^{2}\right)-\Lambda a^{3}-\dot{a}^{2} a+a\right] d t,
$$

where

$$
\mathrm{A}_{k}=\sqrt{8 G / 3} \mathrm{~A}_{k}(t) ; \quad \Lambda=8 \pi G \bar{\Lambda} / 3 .
$$

These redefinitions are made to get $G$ and $\pi$ only in front of the integral, and the integrand to be free of both constants.

In this formula, the point denotes the derivative with respect to $t$, and one supposes that all $k \gg 1$, i.e., we assume that waves with wavelength comparable to the diameter of the Universe are negligible for the dynamics.

The waves packet $\left(\sum_{k}\right)$ ensures the homogeneity of the model during expansion with a high accuracy. After introduction of generalized momenta the Hamiltonian function can be presented as

$$
H=-\frac{P_{a}^{2}}{4 a}-a+\Lambda a^{3}+\sum_{k}\left(\frac{q_{k}^{2}}{4 a^{3}}+k^{2} \mathrm{~A}_{k}^{2} a+m^{2} a^{3} \mathrm{~A}_{k}^{2}\right)=0 .
$$

Here $P_{a}, a$ are a generalized momentum and a coordinate for the Universe as entire; $q_{k}$ and $A_{k}$ are the same for each radial mode of the field. Corresponding to eq. (6) the Wheeler-DeWitt equation for the wave function of the Universe (WF) has the form

$$
\frac{\partial^{2} \Psi}{\partial a^{2}}-\frac{1}{a^{2}} \sum_{k} \frac{\partial^{2} \Psi}{\partial \mathrm{A}_{k}^{2}}-V \Psi=0,
$$

where the superpotential $V$ is given as

$$
V=4\left[a^{2}-\frac{p}{8 a^{2}}\left(1-\frac{p}{2}\right)-\sum_{k} k^{2} \mathrm{~A}_{k}^{2} a^{2}-\Lambda a^{4}\right] .
$$

Here we are neglecting $\sum_{k} m^{2} \mathrm{~A}_{k}^{2} a^{4}$ in comparison with the sum residual in eq. (8) since $k \gg 1$. This is justified because in the short-wavelength approximation we are applying here, the mass of the particles does not have much influence.

The factor ordering parameter $p$ [1] is also introduced in eq. (8).

\section{Semiclassical approximation}

We are going to search a solution of equation (7) in semiclassical approximation

$$
\Psi_{c}=\exp \left(i S_{c}\right)
$$

where $S_{c}$ is a classical action. In this approximation we have

$$
\left(\frac{\partial S_{c}}{\partial a}\right)^{2}-\frac{1}{a^{2}} \sum_{k}\left(\frac{\partial S_{c}}{\partial \mathrm{A}_{k}}\right)^{2}+V=0
$$


This nonlinear equation of the first order is similar to the Hamilton-Jacobi equation of analytical mechanics. For its solution one can take advantage of the complete integral [2]. It can be found from the system of characteristic equations [3]. As the eq. (10) supposes the solution for $(\partial S / \partial a)$, the scale factor becomes an argument along the characteristics. From here we have

$$
\frac{d S_{c}}{d a}=-\frac{V}{F} ; \quad \frac{d \mathrm{~A}_{k}}{d a}=-\frac{q_{k}}{a^{2} F} ; \quad \frac{d q_{k}}{d a}=\frac{4 k^{2} \mathrm{~A}_{k} a^{2}}{F} .
$$

Here $V$ is indicated by (8), and

$$
F=-\sqrt{\left(\sum_{k} q_{k}^{2}\right) / a^{2}-V} .
$$

Now the evolution of the WF $\Psi$ goes along a characteristic. Turning points are the roots of $V(a)=0$. They separate classically available and forbidden regions along the characteristic eq. (11) 四. A similar approach for the strongly anisotropic WF in a Bianchi type I model is presented in ref. [5], and for a closed Friedmann model in ref. [6].

In the region under the barrier the semiclassical solution can be found by an ansatz of the form

$$
\Psi=\exp \left(-\left|S_{e}\right|\right)
$$

The equation for $S_{e}$ can be obtained from eq. (10) by changing $V \rightarrow-V$. Thus, as distinct from eq. (11) the system of the characteristics has the form

$$
\begin{aligned}
\frac{d S_{e}}{d a}=\frac{V}{F_{e}} ; \quad \frac{d \mathrm{~A}_{k}}{d a} & =-\frac{q_{k}}{a^{2} F_{e}} ; \quad \frac{d q_{k}}{d a}=-\frac{4 k^{2} \mathrm{~A}_{k} a^{2}}{F_{e}} ; \\
F_{e} & =-\sqrt{\left(\sum_{k} q_{k}^{2}\right) / a^{2}+V} .
\end{aligned}
$$

\section{The evolution of the scalar field modes in the clas- sically allowed region}

Let us investigate how amplitudes of short wavelength change at the above mentioned evolution of the WF. Equations for $q_{k}$ and $\mathrm{A}_{k}$ follow from (11)

$$
\frac{d \mathrm{~A}_{k}}{d a}=-\frac{q_{k}}{a^{2} F} ; \quad \frac{d q_{k}}{d a}=\frac{4 k^{2} \mathrm{~A}_{k} a^{2}}{F},
$$

where

$$
F=-\sqrt{\left(\sum_{k} q_{k}^{2}\right) / a^{2}-V} .
$$

This system is an essentially non-linear one since co-factors of $q_{k}$ and $\mathrm{A}_{k}$ in the right hand sides of the equations depend on $a$, too. However, the presence of the large parameter $k^{2}$ allows finding a solution.

Following from the system (14), the equation for the generalized momenta $q_{k}$ is

$$
\frac{d^{2} q_{k}}{d a^{2}}=-\frac{4 k^{2} q_{k}}{F^{2}}+\left(\frac{2}{a}-\frac{F^{\prime}}{F}\right) \frac{d q_{k}}{d a},
$$

where $^{\prime}=d / d a$. Here $F(a)$ is an unknown function according to eq. (14). 
It is easy to show that the following solution

$$
q_{k}=\frac{C_{k 0} a}{\sqrt{2 k}} \cos \Phi(a) ; \quad \Phi(a)=2 k \int_{0}^{a} \frac{d a}{F}
$$

satisfies the last equation with the high accuracy of $\mathrm{O}\left(1 / k^{2}\right)$. And correspondingly,

$$
\mathrm{A}_{k}=-\frac{C_{k 0}}{2 \sqrt{2} a k^{3 / 2}} \sin \Phi(a)
$$

where $C_{k 0}$ is an arbitrary constant, and the phase of the solution is chosen from the requirement of amplitude finiteness at $a \rightarrow 0$. The mentioned accuracy of solutions requires the assumption that the logarithmic derivative $F^{\prime} / F$ does not give a large parameter $k$. The last statement follows from the fact that each mode of wave enters into eq. (14) for $F$ as a term

$$
\frac{q_{k}^{2}}{a^{2}}+4 k^{2} \mathrm{~A}_{k}^{2} a^{2}=\frac{C_{k 0}^{2}}{2 k}=\text { const. }
$$

It is clear from substitution eqs. (16), (17) in (18). With taking into account (18) after rewriting of the amplitude $C_{k 0} / \sqrt{2 k}=C_{k}$, we have for $F$ the following expression

$$
F=-\sqrt{\sum_{k} C_{k}^{2}-4\left[a^{2}-\Lambda a^{4}-\frac{p}{8 a^{2}}\left(1-\frac{p}{2}\right)\right]},
$$

Further, we suppose that $\Lambda \ll 1$ and we have in the superpotential

$$
V \simeq 4\left[a^{2}-\frac{p}{8 a^{2}}\left(1-\frac{p}{2}\right)-\frac{1}{4} \sum_{k} C_{k}^{2} \sin ^{2} \Phi(a)\right] .
$$

Values of $V$ and $F$ define an action of WF $\Psi(a)$ according to the system of characteristics (11). Because of the large number of short waves, the sum in eq. (20) can be taken as fast oscillations average with an adequate accuracy. Then the expression for the superpotential gets the form

$$
V=4\left[a^{2}-\frac{p}{8 a^{2}}\left(1-\frac{p}{2}\right)-\frac{1}{8} \sum_{k} C_{k}^{2}\right],
$$

The existence of an inner classically available region requires a realization of the condition $V(a)<0$ in the mentioned interval $0 \leq a \leq a_{0}$. (Let us remember - see eq. (6) - that the total energy $E$ vanishes for the closed universe.) The potential barrier should begin at $a=a_{0}$, i. e. $V(a)>0$ at $a \geq a_{0}$. Let us notice that the vanishing of the factor ordering parameter $(p=0)$ allows us to get an inner classically available region only at the expense of the sum $\sum C_{k}^{2}$. But in that case, a potential barrier necessary for a further development is absent. So, we choose the factor ordering $p=1$ in the following.

Owing to the amplitude of short waves begin to increase quickly under the barrier, we assume that their total energy is negligible in the considered region $V(a)<0$, and the evolution of the Universe is determined only by the first two terms in the r.h.s. of eq. (21). In this case the dynamics of the amplitudes of the short waves can be given explicitly. The phase in eq. (16) is

$$
\Phi(a)=\frac{k}{2} \arcsin \left(\sqrt{1-\left(\frac{a}{a_{0}}\right)^{4}}\right) .
$$


Here, $a_{0}$ can be obtained from the condition

$$
V\left(a_{0}\right)=4\left[a_{0}^{2}-\frac{1}{16 a_{0}^{2}}\right]=0, \quad a_{0}=\frac{1}{2} .
$$

A case when the total energy of the modes is not negligible in the expressions for $V$ and $F$ can be similarly considered. But then the final expression becomes essentially complicated.

\section{Modes of the scalar field under the barrier}

The superpotential $V$ is positive at $a>a_{0}$. This means that the evolution of the WF occurs under the barrier. At this range, the problem is defined by the system of characteristics (13), and we have for $q_{k}$ the following equation

$$
\frac{d^{2} q_{k}}{d a^{2}}=\frac{4 k^{2} q_{k}}{F_{e}^{2}}+\left(\frac{2}{a}-\frac{F_{e}^{\prime}}{F_{e}}\right) \frac{d q_{k}}{d a}
$$

This equation has an inverse sign at first term on the right in comparison with eq. (15) for the classically allowed region. It changes the solution in principle, but for a large parameter $k^{2}$ we can find a solution by a method similar to the one used for eq. (15)

$$
\begin{gathered}
q_{k}=C_{k} a \cosh \Phi_{e}(a) ; \\
\mathrm{A}_{k}=\frac{C_{k}}{2 a k} \sinh \Phi_{e}(a) ; \\
\Phi_{e}(a)=2 k \int_{a_{0}}^{a} \frac{d a}{F_{e}} .
\end{gathered}
$$

Here the conditions of continuity of $q_{k}$ and $\mathrm{A}_{k}$ at the point $a=a_{0}$ were used for the selection of the constants of integration. Both in section 3 and here, the correctness of the solution (25) is determined by the requirement of $F_{e}^{\prime} / F_{e} \ll k$. This requirement is checked by substituting of the obtained solution in $F_{e}$ of eq. (13). There

$$
F_{e}=-\sqrt{\sum_{k} C_{k}^{2}+4 a^{2}} .
$$

In the last formula the term containing the factor ordering, that plays a part only close to zero, is ignored.

Because of the smallness of the sum $\sum_{k} C_{k}^{2}$ under the barrier this term can be neglected in the expression for $F_{e}$, and we suppose with a necessary accuracy

$$
F_{e}(a) \simeq-2 a .
$$

At that $a$ the phase $\Phi_{e}$ has the form

$$
\Phi_{e}=-k \ln \left(\frac{a}{a_{0}}\right),
$$

and leaving only growing modes in solutions at $a>a_{0}$ we obtain

$$
q_{k}=\frac{C_{k} a}{2}\left(\frac{a}{a_{0}}\right)^{k}, \quad \mathrm{~A}_{k}=-\frac{C_{k}}{4 k a}\left(\frac{a}{a_{0}}\right)^{k}
$$




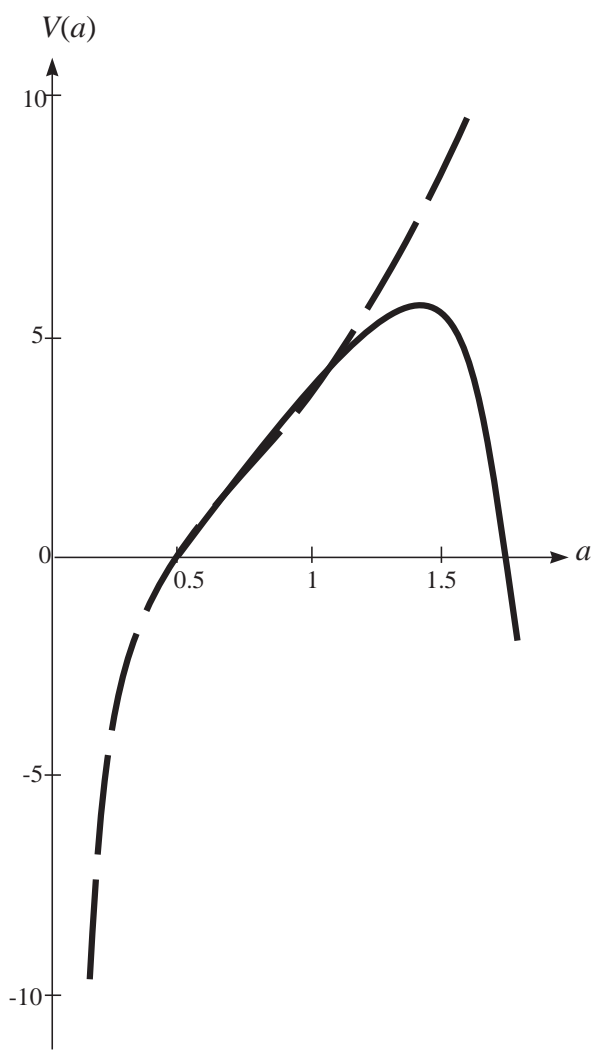

Figure 1: Form of the potential barrier without modes influence (dashed line) and with taking them in account (solid line).

In that solution the amplitude of short waves catastrophically increases with growth of $k$ but not under the exponential law. The latter is realized in the case of a slow variation of $F_{e}$ in eq. (25). It is similar to the Rubakov-effect of catastrophic particle creation, see ref. [7].

A final value of an amplification of the amplitude of short waves is determined by a "time" of the WF stay under the barrier. Let us notice that we consider a self-consistent problem of amplification of short waves (an analogue of particle creation). It means that the energy of the increasing modes under the barrier controls the form of the barrier itself (see Fig. 1).

Let us consider the equation for the action of the WF under the barrier (13)

$$
\frac{d S_{e}}{d a}=\frac{V}{F_{e}} ; \quad F_{e} \simeq-2 a ; \quad V=4 a^{2}-\frac{C_{k}^{2}}{4}\left(\frac{a}{a_{0}}\right)^{2 k} .
$$

Only a "potential" energy of increasing modes is present in the superpotential $V$, but here again it is not cancelled by their "kinetic" energy as it was realized in (26).

As it follows from $V\left(a_{0}, a_{1}\right)=0$, an external boundary of the tunneling region is determined by the equation

$$
V=4 a_{1}^{2}-\frac{C_{k}^{2}}{4}\left(\frac{a_{1}}{a_{0}}\right)^{2 k} .
$$

The final value of amplification of the amplitude of the modes is defined by substitution of $a_{1}$ in eq. (27).

Further, the Euclidean action $S_{e}\left(a_{1}\right)$ has a form

$$
S_{e}\left(a_{1}\right)=\frac{1}{16} \sum_{k} C_{k}^{2}\left(\frac{a_{1}}{a_{0}}\right)^{2 k}\left(\frac{1}{k}-1\right) .
$$


Subject to $k \gg 1$ we have $S_{e}=-a_{1}^{2}$. Hence, a probability of tunneling of the WF through the barrier is given as

$$
w=\exp \left(-2\left|S_{e}\right|\right)=\exp \left(-2 a_{1}^{2}\right)
$$

at $w \ll 1$. The formula

$$
w=\frac{\exp \left(-2 a_{1}^{2}\right)}{1+\exp \left(-2 a_{1}^{2}\right)}
$$

is more exact in the case $w<1$.

\section{Conclusions}

The self-consistent problem of the evolution of the closed Friedmann Universe with an amplification of wave perturbations of a scalar field under a barrier is considered. Within the framework of a semiclassical approximation of the Wheeler-DeWitt equation it is shown that the increase of the amplitude of short waves under the barrier controls the barrier shape, i.e. leads to both a reduction of the barrier and fast output of WF from under the barrier. In this sense the problem is self-consistent. The process of tunneling and the increase of the amplitudes of short waves is defined here by the assignment of their initial spectrum in the inner classically allowed region. If it is required after tunneling in the Universe to receive a spectrum close to the flat one, the initial spectrum should be almost exponentially suppressed in the area $k \rightarrow \infty$. It is qualitatively equivalent to the presence of an effective temperature in the inner classically allowed region.

A more detailed discussion of these problems obviously requires a consideration of the quantum nature of the problem for short-wave perturbations of a scalar field.

\section{ACKNOWLEDGEMENT}

The authors thank A. A. Starobinsky and M. V. Sazhin for information about papers of V. A. Rubakov et al. and H. Kleinert for useful comments. H.-J.S. acknowledges financial support from the HSP III-program. The work is done within the project KR-154 of the International Science and Technology Centre (ISTC).

\section{References}

[1] A. D. Linde, "Elementary Particle Physics and Inflationary Cosmology", Nauka, Main Editorial Board for Physical and Mathematical Literature, Moscow, 1990.

[2] L.D. Landau, E.M. Livshits, "Mechanics". Nauka, Main Editorial Board for Physical and Mathematical Literature, Moscow, 1988.

[3] E. Kamke, "The reference book on differential partial equations of the first order". Nauka, Moscow, 1966.

[4] V. Ts. Gurovich, U. M. Imanaliev and I. V.Tokareva, JETP Lett 64, no.5, 329 (1996).

[5] M. Bachmann and H.-J. Schmidt, gr-qc/9912068, Phys. Rev. D in press.

[6] V. Folomeev and V. Gurovich, Gen. Rel. Grav. 32 (2000) in press.

[7] V.A. Rubakov, Pis'ma Zh.Eksp. Teor. Fiz 39, No. 2, 89-92 (1984). G.V. Lavrelashvili, V.A. Rubakov and P.G. Tenyakov, Nuclear Physics B299, No. 2, 757-796 (1988). 